Analysis of nickel concentration profiles around the roots of the hyperaccumulator plant Berkheya coddii using MRI and numerical simulations

\author{
Journal Article \\ Author(s): \\ Moradi, A.B.; Oswald, S.E.; Nordmeyer-Massner, J.A.; Prüssmann, Klaas P.; Robinson, B.H.; Schulin, R. \\ Publication date: \\ 2010 \\ Permanent link: \\ https://doi.org/10.3929/ethz-b-000017112 \\ Rights / license: \\ In Copyright - Non-Commercial Use Permitted \\ Originally published in: \\ Plant and Soil 328(1-2), https://doi.org/10.1007/s11104-009-0109-8
}




\title{
Analysis of nickel concentration profiles around the roots of the hyperaccumulator plant Berkheya coddii using MRI and numerical simulations
}

\author{
A. B. Moradi • S. E. Oswald • \\ J. A. Nordmeyer-Massner • K. P. Pruessmann • \\ B. H. Robinson $\cdot$ R. Schulin
}

Received: 8 April 2009 / Accepted: 11 July 2009/Published online: 25 July 2009

(C) Springer Science + Business Media B.V. 2009

\begin{abstract}
Investigations of soil-root interactions are hampered by the difficult experimental accessibility of the rhizosphere. Here we show the potential of Magnetic Resonance Imaging (MRI) as a non-destructive measurement technique in combination with numerical modelling to study the dynamics of the spatial distribution of dissolved nickel $\left(\mathrm{Ni}^{2+}\right)$ around the roots of the
\end{abstract}

Responsible Editor: Henk Schat.

A. B. Moradi $(\varangle) \cdot$ B. H. Robinson $\cdot$ R. Schulin

Soil Protection group, Institute of Terrestrial Ecosystems,

ETH Zurich,

CHN F28.2, Universitaetstrasse 16,

CH-8092 Zurich, Switzerland

e-mail: ahmad.moradi@env.ethz.ch

S. E. Oswald

Hydrogeology Department, Helmholtz Centre

for Environmental Research-UFZ,

Permoserstraße 15,

04318 Leipzig, Germany

\section{J. A. Nordmeyer-Massner - K. P. Pruessmann} Institute for Biomedical Engineering,

University and ETH Zurich,

Zurich, Switzerland

Present Address:

A. B. Moradi

Hydrogeology Department, Helmholtz Centre

for Environmental Research-UFZ,

Permoserstraße 15,

04318 Leipzig, Germany

e-mail: ahmad.moradi@ufz.de

URL: http://www.ufz.de/index.php?de=17424 nickel hyperaccumulator plant Berkheya coddii. Special rhizoboxes were used in which a root monolayer had been grown, separated from an adjacent inert glass bead packing by a nylon membrane. After applying a $\mathrm{Ni}^{2+}$ solution of $10 \mathrm{mg} \mathrm{l}^{-1}$, the rhizobox was imaged repeatedly using MRI. The obtained temporal sequence of 2-dimensional $\mathrm{Ni}^{2+}$ maps in the vicinity of the roots showed that $\mathrm{Ni}^{2+}$ concentrations increased towards the root plane, revealing an accumulation pattern. Numerical modelling supported the $\mathrm{Ni}^{2+}$ distributions to result from advective water flow towards the root plane, driven by transpiration, and diffusion of $\mathrm{Ni}^{2+}$ tending to eliminate the concentration gradient. With the model, we could study how the accumulation pattern of $\mathrm{Ni}^{2+}$ in the root zone transforms into a depletion pattern depending on transpiration rate, solute uptake rate, and $\mathrm{Ni}^{2+}$ concentration in solution.

Keywords Berkheya coddii . Concentration gradient . Hyperaccumulator - Magnetic resonance imaging · MIN3P $\cdot$ Modelling $\cdot$ Nickel $\cdot$ Uptake

\section{Introduction}

The focus of rhizosphere studies has recently been opened to the fate of soil pollutants such as heavy metals as a consequence of increasing awareness of soil pollution problems and the attempts to control the pollutants and to remediate contaminated soils 
(Wenzel 2005). Nickel is a heavy metal of concern in soil that can be of geogenic origin, as in serpentine soils (Robinson et al. 1999), or may arise from anthropogenic pollution (Brooks 1998; Ashworth and Alloway 2004). Nickel hyperaccumulator plants growing on serpentine soils are able to accumulate at least ten times more nickel than other plant species growing in the same environment (Brooks et al. 1977). Therefore, some hyperaccumulator plants may offer a sustainable treatment option for the remediation of metal-contaminated sites, and an opportunity to mine naturally metal-rich soils by phytomining (Brooks et al. 1998; Li et al. 2003). An attractive plant for phytoextraction of Ni is Berkheya coddii Roessler. Its extraordinary phytoextraction potential results from the rare combination of high biomass $\left(22 \mathrm{tha}^{-1}\right)$, and high capacity of $\mathrm{Ni}$ accumulation in its above-ground biomass which can reach up to $1 \% \mathrm{w}$ : (Robinson et al. 1997). Despite this, little is known about the mechanism of $\mathrm{Ni}$ hyperaccumulation and root-soil interactions of $\mathrm{Ni}$ hyperaccumulator plants including Berkheya coddii. Understanding these mechanisms and interactions could help improving methods for the phytoextraction of $\mathrm{Ni}$ from contaminated soils and also phytomining (McNear et al. 2005).

However, investigating root-soil interactions such as the uptake of metals by roots is challenging. It requires a high-resolution data on the spatial distribution of solutes because these processes create substantial differences in concentrations over distances of a few millimetres from root surfaces. Many rhizosphere processes are dynamic (Darrah 1993; Gregory and Hinsinger 1999; Wenzel et al. 2001; Hinsinger et al. 2006), therefore a non-destructive, non-invasive method to observe rhizosphere processes resolved in space and time is desirable.

The uptake of trace metals, such as nickel, by roots, is generally considered to occur through more or less specific membrane transporters (Zhao et al. 2002; Whiting et al. 2003). If a metal is removed by roots from the rhizosphere more rapidly than it can be resupplied, by bulk soil via desorption and diffusion, the metal will become depleted adjacent to the root surface (Tinker and Nye 2000). In contrast, if the mass flow of a solute from the bulk soil towards the roots exceeds the uptake rate of the solute, then a concentration gradient opposing the advection flux will develop. The resulting concentration profiles are further influenced by chemical reactions with other compounds in the rhizosphere and with the soil matrix. Although these processes in rhizosphere have been discovered earlier, but due to technical limitations, they have never been observed in situ with sufficient temporal and spatial resolution. Combining new non-destructive measurement techniques such as MRI and numerical simulations can unravel some aspects of these complex interactions.

Among other new techniques, Magnetic Resonance Imaging (MRI) has been proposed to assess the spatial distribution of water and metal ions in porous media (Herrmann et al. 2002; Pierret et al. 2003; Moradi et al. 2008). The presence of paramagnetic ions and molecules in a solution affects the spin vector relaxation times (longitudinal relaxation time $T_{1}$ and transverse relaxation time $T_{2}$ ) of water protons $\left({ }^{1} \mathrm{H}\right)$. The determination of these relaxation times provides opportunities to trace the movement and diffusion of dissolved paramagnetic compounds and ions such as $\mathrm{Ni}^{2+}$ in the porous media. However, applying MRI to soil-plant system has several limitations. The presence of ferromagnetic particles and other paramagnetic compounds in soils can distort the MRI images and cause signal loss. Therefore, at the present state, this technique mainly is applied to carefully selected media such as pretreated sand and soil, agar, and glass beads.

In a previous study we developed a MRI method to study the temporal and spatial distribution of dissolved $\mathrm{Ni}^{2+}$ ions in a porous medium under the influence of absorption by an exchange resin (Moradi et al. 2008). Here we showed that MRI can detect concentration profiles and thus can be used to investigate $\mathrm{Ni}^{2+}$ uptake by hyperaccumulator plants in a more complex system. We studied the twodimensional spatial and temporal distribution of $\mathrm{Ni}^{2+}$ from a planar monolayer mat of roots of the hyperaccumulator plant Berkheya coddii. We also used numerical modelling to interpret the measurements and to analyse the dependence of the $\mathrm{Ni}^{2+}$ gradient adjacent to the roots on transpiration rate, uptake rate and initial concentration of $\mathrm{Ni}^{2+}$.

\section{Materials and methods}

Rhizobox setup and plant growth

The rhizoboxes (inner dimensions, $100 \times 100 \times$ $30 \mathrm{~mm}$ ) used in this study (Fig. 1) were a modified 
Fig. 1 The rhizobox set up with a view of the outlet and inlet ports (left) and the root-only compartment (right)
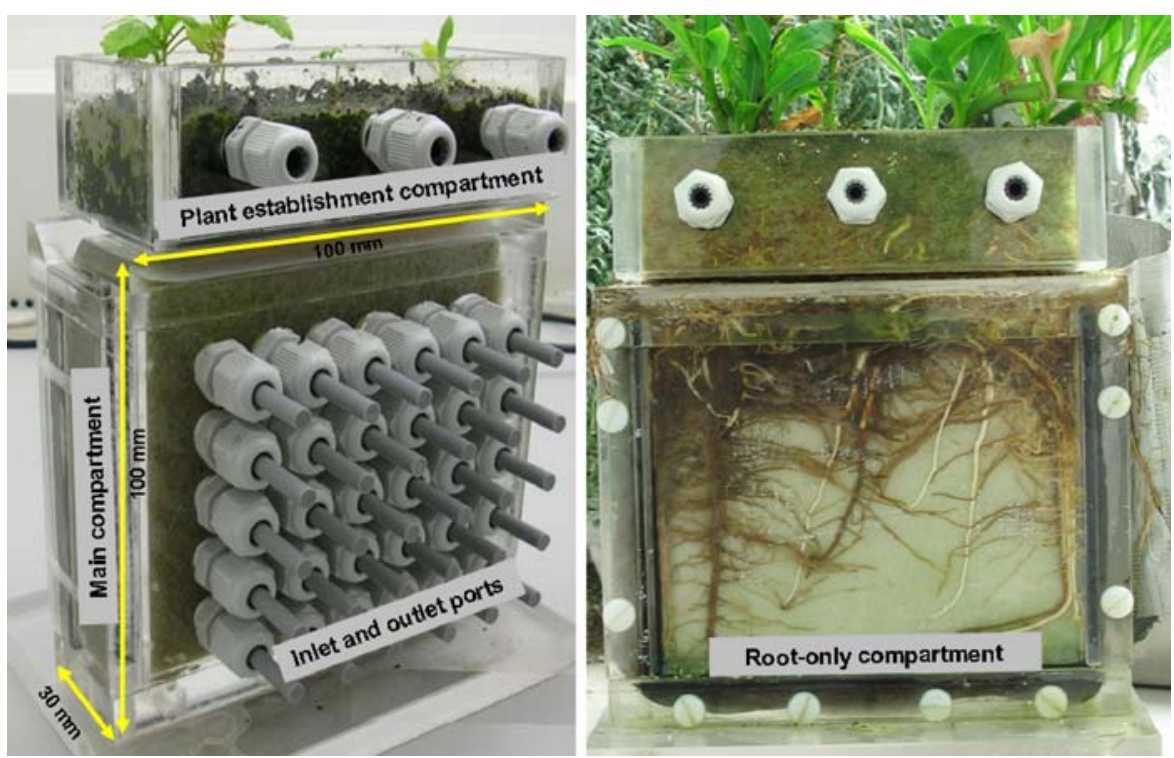

version of the rhizobox system developed and tested by Wenzel et al. (2001). We changed the frame of the rhizobox so that the MRI receiver coils could be installed close to the root plane on the transparent side of the rhizobox (an acrylic window for root visualization and root growth control) to obtain maximal coil sensitivity in the volume of interest. We sealed the rhizoboxes using rubber bands and silicon glue. The experiment started from fully water-saturated condition, which gave the strongest MRI signal-to-noise ratio (SNR). We also redesigned the inlet and outlet ports and increased their number to 30 per box, arranged on one side of the box in five rows of six ports each, in order to improve the regulation of the nutrient solution in the main compartment of the rhizobox.

Seeds of Berkheya coddii were pre-germinated in perlite and grown until they were 2 weeks old. The seedlings were transferred to the upper compartment of the rhizoboxes. The main and the upper compartments of each rhizobox were filled with porous glass beads (Sera Werke, Germany) of 0.4-1.0 mm-diameter. The total porosity of the packing was $65 \% \mathrm{v} / \mathrm{v}$, and the bulk density was $0.46 \mathrm{~g} \mathrm{~cm}^{-3}$. Four rhizoboxes were prepared in total and four seedlings were planted per rhizobox. After transplanting, the rhizoboxes were kept for 8 weeks in a climate chamber with a daily light cycle (1.0-1.2 lumen $\left.\mathrm{cm}^{-3}\right)$ of $16 \mathrm{~h}$ light/ $8 \mathrm{~h}$ darkness, constant humidity (75\%) and controlled temperature $\left(23 / 16^{\circ} \mathrm{C}\right.$ day/night). The rhizoboxes were irrigated with nutrient solution (Kraemer et al. 1996). A peristaltic pump was used to circulate the nutrient solution through the rhizoboxes at a rate of around 0.61 per day. The lower two rows of the ports were used as inlets for the nutrient solution and the ports of the uppermost row were used as outlets. Roots started to grow into the root-only compartment a week after transplanting. The root-only compartment contained no glass beads or soil. It consisted of a narrow slit separated from the glass beads by a nylon membrane with a mesh size of $20 \mu \mathrm{m}$. After 8 weeks of growth, one of the rhizoboxes that showed the most uniform root plane was chosen and the MRI experiments were performed on this rhizobox.

\section{MRI technique and data analysis}

MRI was performed using a Philips Intera wholebody system (Philips medical systems, Best, the Netherlands) with a static magnetic field of 1.5 Tesla that results in a proton resonance frequency of 63.8 $\mathrm{MHz}$. We used a $T_{1}$ mapping technique known as variable flip angle for the quantification of $T_{1}$ (Deoni et al. 2003; Treier et al. 2007). This method calculates $T_{1}$ with the same accuracy but with a significantly shorter acquisition time than standard $T_{1}$ measurement sequences. It is based on the consecutive application of $T 1$-weighted spoiled gradient-echo (T1 fast-field echo) sequences using different flip 
angles. The steady state signal achieved for a sequence using phase alternated radio-frequency (RF) pulses can be calculated using Eq. 1:

$$
\begin{aligned}
\frac{I(\theta)}{\sin (\theta)}= & \exp \left(-T R / T_{1}\right) \frac{I(\theta)}{\tan (\theta)}+N(H) \\
& \times\left(1-\exp \left(-T R / T_{1}\right)\right) \\
& \times \exp \left(-T E / T_{2}^{*}\right)
\end{aligned}
$$

where $I$ is the signal intensity, $N(H)$ is the proton density, $\theta$ is the $R F$ pulse flip angle, $T E$ is the echo time, and $T_{2}{ }^{*}$ is the effective transverse relaxation time. According to Eq. 1, a plot of $I(\theta) / \sin (\theta)$ vs. $I(\theta) / \tan (\theta)$ should yield a straight line with slope $\exp \left(-T R / T_{1}\right)$. Since this slope depends only on $T R$, the known repetition time, one can calculate $T_{1}$ without prior knowledge of $N(H)$ or $T_{2}{ }^{*}$. The effect of transverse relaxation was considered negligible for a fairly short echo times. We used an echo time of $4.5 \mathrm{~ms}$, a repetition time of $9 \mathrm{~ms}$, slice thickness of $15 \mathrm{~mm}$, a data matrix of $512 \times 512$ pixels, and a field of view of $110 \times 110 \mathrm{~mm}$. The acquisition bandwidth was $564.10 \mathrm{~Hz}$. For $T_{1}$ calculation, we cropped the image part related to the rhizobox in the middle of the original MRI matrix, therefore the images shown here are only the cross-sections of the rhizobox. We fitted the Eq. 1 to the MRI data for different flip angles and calculated the $T_{1}$ for each pixel. Due to the limited spatial sensitivity of the receiver coil, the SNR deteriorated on the two sides of the rhizobox due to the higher distance from the coil centre. To avoid measurement errors due to partial volume effects we excluded a margin of $5 \mathrm{~mm}$ width from the two sides of the MRI images for further analysis (Fig. 3b). A median filter with dimensions of $3 \times 3$ pixels was applied to the $T_{1}$ images to reduce noise. All the images were processed using Matlab.

In a separate experiment, we found a linear relationship between the longitudinal relaxation time, $T_{1}$, and the $\mathrm{Ni}^{2+}$ concentrations in glass beads saturated with $\mathrm{Ni}^{2+}$ solution in the concentration range of $1-30 \mathrm{mg} \mathrm{l}^{-1}$ (Fig. 2). This calibration curve was used for calculating $\mathrm{Ni}^{2+}$ concentrations for each pixel from the corresponding $T_{1}$ values.

$\mathrm{Ni}^{2+}$ uptake experiment

For the $\mathrm{Ni}^{2+}$ uptake experiment, the main compartment of the rhizobox was washed with eight pore-

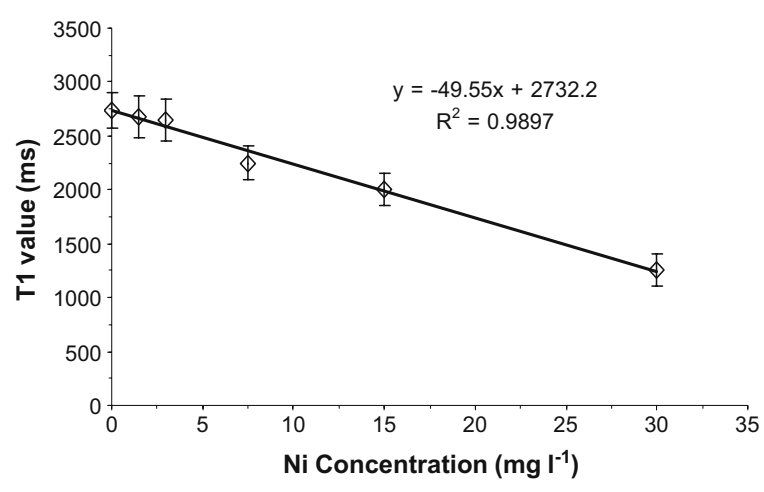

Fig. 2 The calibration of $T_{1}$ versus $\mathrm{Ni}^{2+}$ concentration; bars represent standard deviations of the mean

volumes of $\mathrm{Ni}^{2+}$ solution with a concentration of $10 \mathrm{mg}^{-1}$ (prepared using $\left.\mathrm{Ni}\left(\mathrm{NO}_{3}\right)_{2}, 6 \mathrm{H}_{2} \mathrm{O}\right)$ to replace the nutrient solution. We used the lower ports as inlets for ingoing $\mathrm{Ni}^{2+}$ solution and let the solution overflow over the lateral walls of the rhizobox to ensure homogeneous initial $\mathrm{Ni}^{2+}$ distribution in the system. This rather high concentration of $\mathrm{Ni}^{2+}$ was used to stay clearly above the detection limit for $\mathrm{Ni}^{2+}$ of $1 \mathrm{mg} 1^{-1}$. The rhizobox was put into the MRI scanner (Fig. 3a) and a circular RF coil was installed on the root-plane side of the rhizobox close to the roots. The first $T_{1}$ measurement could be carried out $3 \mathrm{~h}$ after the $\mathrm{Ni}^{2+}$ solution was applied to the rhizobox. The second and the third measurement were performed 5 and $11 \mathrm{~h}$ after the application of $\mathrm{Ni}^{2+}$ solution, respectively. The amount of transpiration was measured by weighing the rhizobox before and after the experiment. We kept the upper compartment dry for a week before the MRI experiment started, therefore, evaporation during the experiment and the transpiration from the upper compartment was considered negligible and the water was assumed to be taken up by plant roots only from the main compartment of the rhizobox.

\section{Modelling}

Simulations were carried out using the numerical code MIN3P (Mayer et al. 2002), which describes variablysaturated flow and transport in three-dimensional porous media and geochemical reactions including kinetic and equilibrium mass exchange with mineral phases and roots. The simulation of variably saturated water flow is described by the Richards equation, 

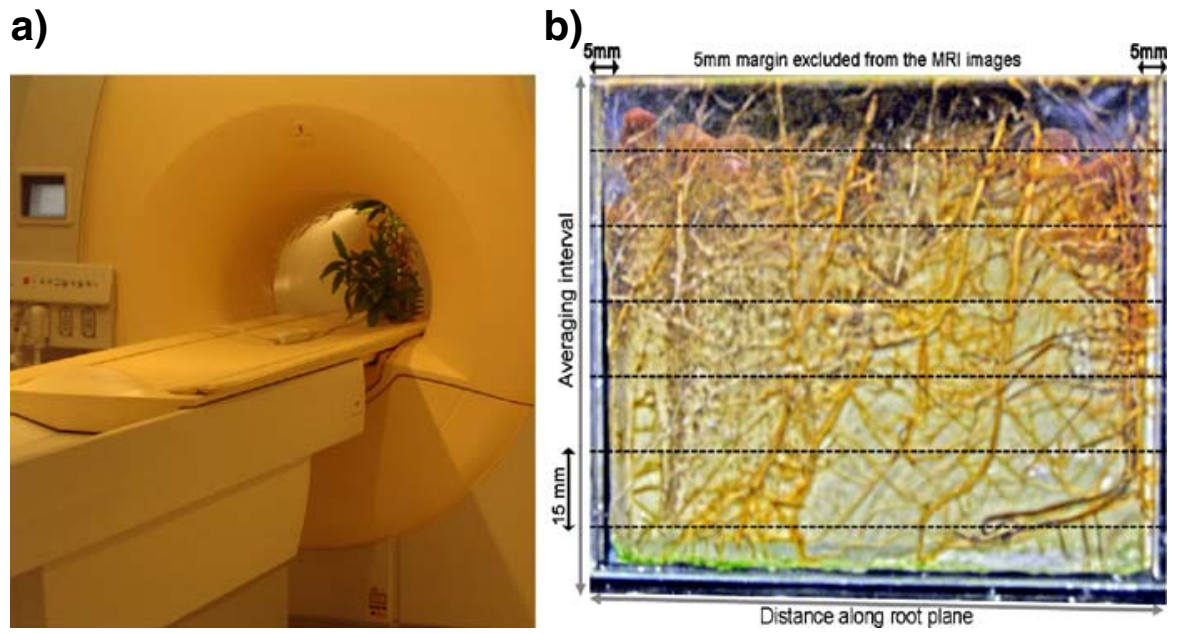

Fig. 3 A view of the rhizobox placed inside the MRI machine (a) and the root distribution pattern before the $\mathrm{Ni}^{2+}$ uptake experiment (b). The broken lines indicate the $15 \mathrm{~mm}$ thick slices into which MRI signal was recorded

which is implemented in MIN3P using the formulation (Mayer et al. 2002):

$S_{a} S_{s} \frac{\partial h}{\partial t}+\phi \frac{\partial S_{a}}{\partial t}-\nabla \cdot\left[k_{r a} K \nabla h\right]-Q_{a}=0$

Where $t$ is time (s), $\phi$ is the total porosity, $S_{a}$ is water saturation, $S_{s}$ is the specific storage coefficient $\left(\mathrm{m}^{-1}\right), Q_{a}$ is the sink/source term, $K$ is the hydraulic conductivity tensor $\left(\mathrm{m} \mathrm{s}^{-1}\right), k_{r a}$ is the relative permeability of the porous medium with respect to the aqueous phase and is related to the hydraulic head, $h$, and water saturation, $S_{a}$, through the standard soil hydraulic functions given by Wosten and Vangenuchten (1988).

The mass balance equation for the reactive transport of the solutes was as followings:

$$
\begin{aligned}
\frac{\partial}{\partial t}\left(S_{a} \phi T_{j}^{a}\right)= & \nabla \cdot\left(S_{a} \phi D_{a} \nabla T_{j}^{a}\right)-\nabla \cdot\left(v T_{j}^{a}\right) \\
& -R_{j}-q T_{j}^{a},
\end{aligned}
$$

Where $T_{j}^{a}$ is the total aqueous concentration of the component $j\left(\mathrm{~mol} \mathrm{~m}{ }^{-3}\right), \nu$ is the Darcy velocity $\left(\mathrm{m} \mathrm{s}^{-1}\right)$, $D_{a}$ is the hydrodynamic dispersion coefficient $\left(\mathrm{m}^{2} \mathrm{~s}^{-1}\right)$, $R j$ is the sink/source term for kinetically-controlled reactions, and $q$ is the water uptake flux $\left(\mathrm{s}^{-1}\right)$.

We used MIN3P to simulate $\mathrm{Ni}^{2+}$ distribution in the rhizobox and adjacent to the root plane as being taken up by roots. The simulation was set to start with the initial condition of water saturated for the rhizobox and no flux from the boundaries except for the root plane that was assumed to be a homogeneous sink for water and $\mathrm{Ni}^{2+}$ Soil hydraulic function parameters were set to following values; residual water content was 0.05 , Van Genuchten $\alpha$ was $0.143 \mathrm{~m}^{-1}$, Van Genuchten $n$ was 1.506 and Van Genuchten $l$ was 0.65 . Water was set to be taken up at a constant rate during the experiment. Nickel uptake was modeled following the approach by Lombi et al. (2001) that adds a linear component to the MichaelisMenten kinetics:

$F_{M}=\frac{k_{m} c}{K_{m}+c}+\alpha c$

where $F_{M}$ is the root uptake rate per unit surface of the roots $\left(\mathrm{mol} \mathrm{m} \mathrm{m}^{-2} \mathrm{~s}^{-1}\right), k_{m}$ is the effective rate coefficient ( $\mathrm{mol} \mathrm{m} \mathrm{m}^{-2} \mathrm{~s}^{-1}$ ), $K_{m}$ is the MichaelisMenten's constant $\left(\mathrm{mol} \mathrm{m}{ }^{-3}\right), c$ is the $\mathrm{Ni}^{2+}$ concentration in solution $\left(\mathrm{mol} \mathrm{m}^{-3}\right)$, and $\alpha$ is the slope of the linear component $\left(\mathrm{mol} \mathrm{m} \mathrm{m}^{-2} \mathrm{~s}^{-1}\right)$.

Sensitivity analysis showed that the most critical parameters were uptake rate coefficient and transpiration rate (data not shown here). The $\mathrm{Ni}^{2+}$ uptake rate coefficient, the Michaelis-Menten's constant and the linear component were varied randomly to yield the observed temporal evolution of the $\mathrm{Ni}^{2+}$ distribution. Root mean square error (RMSE) measure was used to evaluate the simulations and the one with the smallest RMSE was finally chosen. The $\mathrm{Ni}^{2+}$ diffusion coefficient in free aqueous solution was set to $1.25 \times$ $10^{-9} \mathrm{~m}^{2} \mathrm{~s}^{-1}$. The dispersivity was set to $9.0 \times 10^{-4} \mathrm{~m}$, 
which reflects the grain-size of the glass beads we used $(0.4-1.0 \mathrm{~mm})$ and agrees with the typical range of dispersivity in soils (Aggelopoulos and Tsakiroglou 2007). The initial $\mathrm{Ni}^{2+}$ concentration was $10 \mathrm{mg} \mathrm{l}^{-1}$. The other parameters were taken directly from the experiment. The glass beads were chemically inert; therefore, no sorption isotherm was used. The grid resolution for numerical solution was set to correspond to the spatial resolution of the MRI measurement, except that a finer grid was chosen in the direct vicinity of the roots for better convergence with the numerical solution.

\section{Results}

Temporal changes of $\mathrm{Ni}^{2+}$ distribution

Figure $3 \mathrm{~b}$ shows the distribution pattern of roots in the root-only compartment before the $\mathrm{Ni}^{2+}$ uptake experiment started. The horizontal sections, separated by the dashed lines correspond to the slices into which the MRI signals were collected. There was a dense layer of roots in the first two slices, but the root density was lower in the bottom of the rhizobox. The root mat was composed of old and young roots in all slices. The root distribution was most homogeneous in the $3 \mathrm{rd}, 4$ th and 5 th slice from the top. These three slices remained water saturated during the course of experiment, therefore, they were chosen for calculations of $T_{1}$ and $\mathrm{Ni}^{2+}$ concentration profiles.

The $\mathrm{Ni}^{2+}$ distribution maps measured at various times after the application of $\mathrm{Ni}^{2+}$ solution are shown in Fig. 4. Each $\mathrm{Ni}^{2+}$ distribution map represents a pixel-wise average value of the slices 3,4 and 5 that were calculated from the corresponding $T_{1}$ values using the calibration curve in Fig. 2. $T_{1}$ values ranged from $1,800 \mathrm{~ms}$ near the root plane to slightly above $2,400 \mathrm{~ms}$ in the corners of the rhizobox farthest away from the root plane (data not shown here). Compared to the middle of the rhizobox, the corners of the rhizobox were farthest away from the coil centre and showed a weaker SNR and therefore considerably higher variability in the calculated $\mathrm{T} 1$ and the corresponding $\mathrm{Ni}^{2+}$ concentrations. This indicates that the reliability of the measurement decreased with distance from the coil centre due to a decline in SNR. Already at the first measurement, $3 \mathrm{~h}$ after the start of
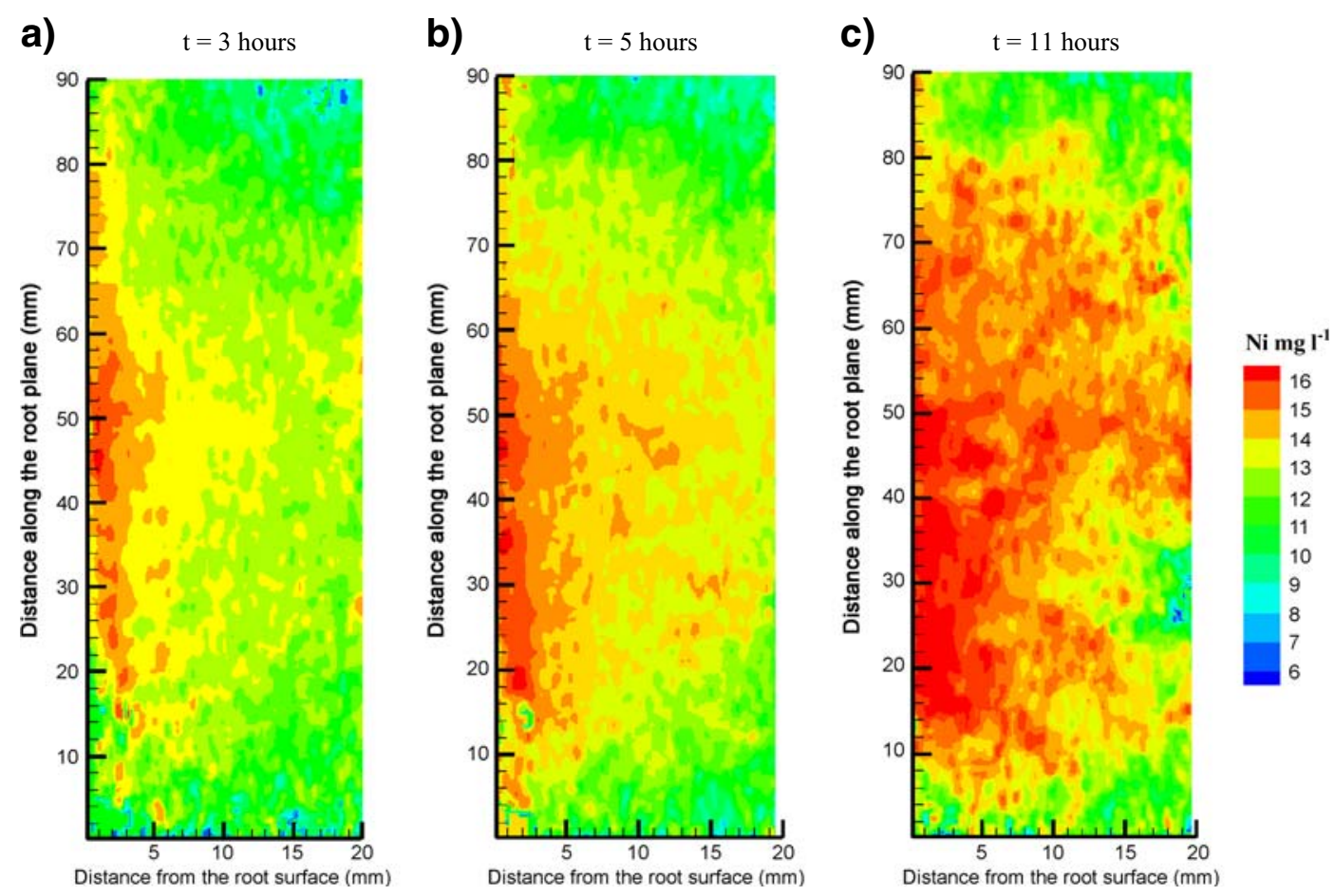

Fig. 4 Horizontal two-dimensional maps of $\mathrm{Ni}^{2+}$ distribution in the root zone of Berkheya coddii $3 \mathrm{~h} \mathrm{(a),} 5 \mathrm{~h}(\mathbf{b})$, and $11 \mathrm{~h}(\mathbf{c})$ after $\mathrm{Ni}^{2+}$ application to the rhizobox 
the experiment (application of $\mathrm{Ni}^{2+}$ solution to the rhizobox), a gradient in the $\mathrm{Ni}^{2+}$ concentration was visible (Fig. 4a). The $\mathrm{Ni}^{2+}$ concentration ranged from around $15 \mathrm{mg} \mathrm{l}^{-1}$ immediately adjacent to the rootplane to slightly above $10 \mathrm{mg}^{-1}$ at a distance of $20 \mathrm{~mm}$ away from the roots on the opposite side of the rhizobox. This indicates that the amount of $\mathrm{Ni}^{2+}$ transported to the root surface via convection was greater than the amount of $\mathrm{Ni}^{2+}$ taken up by the roots. The roots partially excluded the $\mathrm{Ni}^{2+}$ and the extent of the exclusion in the middle of the root-plane was greater than at the edges. This variability might be related to the inhomogeneous distribution and activity of the roots. After $5 \mathrm{~h}$, the $\mathrm{Ni}^{2+}$ accumulation zone extended to a distance of more than $20 \mathrm{~mm}$ away from the root surface in the middle of the root-plane (Fig. 4b). The $\mathrm{Ni}^{2+}$ accumulation zone extended to the sides of the rhizobox $11 \mathrm{~h}$ after the experiment had started (Fig. 4c), while the magnitude of the accumulation was still highest in the middle.

$\mathrm{Ni}^{2+}$ concentration mapping at different soil water contents

As the plants transpired water, the water level in the rhizobox dropped and the upper part of the rhizobox became unsaturated. With the decrease in water content, also the SNR decreased in the upper part of the rhizobox (mainly first and the second slice from top). This resulted in high variability in the calculated $T_{1}$ values. $T_{1}$ maps of horizontal cross-sections through the rhizobox at the upper first slice (unsaturated), the second slice (partly saturated), and the 4th slice (saturated) are shown in Fig. 5a, b, and c, respectively. In the unsaturated zone (Fig. 5a), the SNR was much poorer than in the saturated zone below. Water was the main signal-bearing source in our system, therefore a decrease in water content resulted in decrease in SNR. Since the main aim here was to visualize $\mathrm{Ni}^{2+}$ concentrations close to the roots where full water saturation was persistent during the experiments, we did not further process the MRI data from the unsaturated part of the rhizobox. The SNR increased in the second slice of the rhizobox in the transition zone between saturated and unsaturated zone (Fig. 5b) i.e. in the zone of capillary fringe above the water level, but the signal was considerably lower and more heterogeneous than in the fully saturated zone (Fig. 5c).
Modelling $\mathrm{Ni}^{2+}$ uptake

Figure 6 shows the MRI-measured and the simulated average profiles of $\mathrm{Ni}^{2+}$ concentrations with distance from the root plane for each measurement time. The MRI-measured averages were taken column-wise parallel to the root plane from the $3 \mathrm{rd}$, 4 th, and 5 th slices.

The best agreement between the simulated and MRI-measured $\mathrm{Ni}^{2+}$ concentration profiles (based on RMSE analysis) was obtained when the uptake rate coefficient was set to $1.17 \times 10^{-8} \mathrm{~mol} \mathrm{~m}^{-2} \mathrm{~s}^{-1}$, the Michaelis-Menten's constant to $2.5 \times 10^{-4} \mathrm{~mol} \mathrm{~m}^{-3}$, and the linear component to $1.05 \times 10^{-9} \mathrm{~mol} \mathrm{~m}^{-2} \mathrm{~s}^{-1}$. These values are well in the same order of magnitude with the published rates for zinc and cadmium by other hyperaccumulator plants (Lombi et al. 2001; Zhao et al. 2006). The concentration range of $\mathrm{Ni}^{2+}$ in solution was almost three orders of magnitude higher than the optimized Michaelis-Menten's constant which shows that the $\mathrm{Ni}^{2+}$ uptake by root was mainly passive instead of active. The simulation of the averaged $\mathrm{Ni}^{2+}$ profile at $t=3 \mathrm{~h}$ was the most satisfactory $(\mathrm{RMSE}=0.20$ compared to 0.26 and 0.46 for $t=5$ and 11 respectively). The MRImeasured $\mathrm{Ni}^{2+}$ concentration was in good agreement with the simulated $\mathrm{Ni}^{2+}$ profile over the whole length from the root plane to the opposite wall. For $t=5$ and 11, the simulated $\mathrm{Ni}^{2+}$ concentration profiles were slightly overestimated adjacent to the root surface and underestimated at farther distances namely $5-15 \mathrm{~mm}$ from the roots. The MRI-measured $\mathrm{Ni}^{2+}$ profiles showed a gentler slope than the simulated ones.

Effects of various parameters on $\mathrm{Ni}^{2+}$ concentration profile

The magnitude and the sign of the $\mathrm{Ni}^{2+}$ gradient adjacent to the roots are essentially a function of $\mathrm{Ni}^{2+}$ concentration in solution, the rate of $\mathrm{Ni}^{2+}$ uptake by the roots, and the transpiration rate. We performed a sensitivity analysis by varying each one of these parameters at a time while keeping the other parameters constant. Figure 7 shows how transpiration rate affected the simulated $\mathrm{Ni}^{2+}$ concentration gradient. The transpiration rate was reduced by 45 and $85 \%$, while all other parameters were kept constant. Reduction of the transpiration by $45 \%$ eliminated the gradient profile, while $85 \%$ reduction in transpi- 

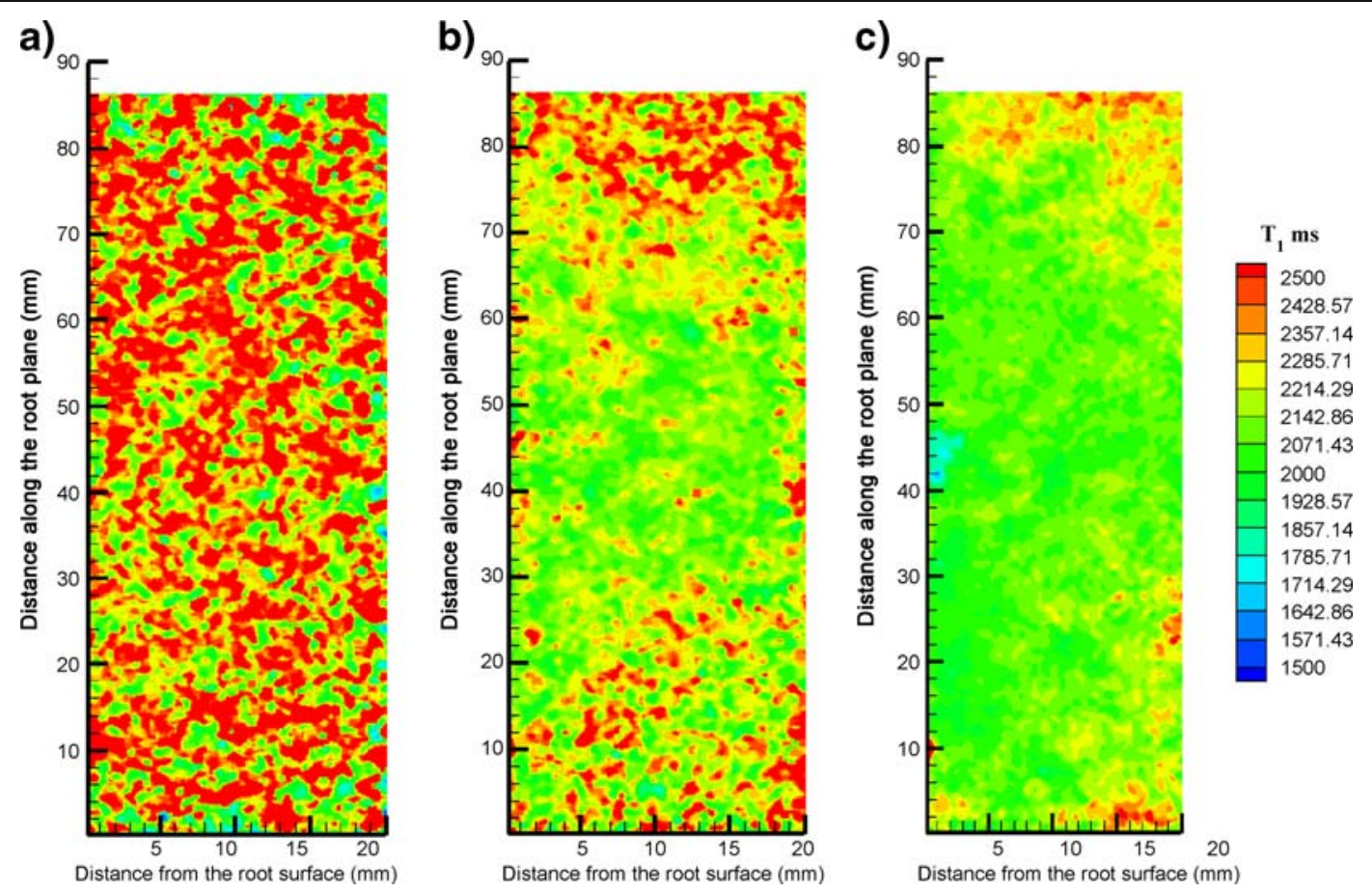

Fig. $5 T_{1}$ maps at horizontal cross-sections of the rhizobox at $t=11 \mathrm{~h}$ when the water level dropped in the rhizobox: a) 1 st slice from top where it was unsaturated, b) 2nd slice partly saturated, and c) 4th slice completely saturated

ration resulted in a negative gradient towards the roots.

Varying the uptake rate coefficient had similar effects on the $\mathrm{Ni}^{2+}$ concentration profile (Fig. 8). The $\mathrm{Ni}^{2+}$ concentration gradient became gentler and then disappeared when the uptake rate coefficient was increased to $2.7 \times 10^{-8}$ and $4.95 \times 10^{-7} \mathrm{~mol} \mathrm{~m}^{-2} \mathrm{~s}^{-1}$ respectively. Using an uptake rate coefficient of $1.62 \times 10^{-6} \mathrm{~mol} \mathrm{~m}{ }^{-2} \mathrm{~s}^{-1}$ resulted in $\mathrm{Ni}^{2+}$ depletion in the rhizosphere.
Fig. 6 The MRI-measured and simulated $\mathrm{Ni}^{2+}$ concentration profiles as a function of distance from the root plane at 3,5 , and $11 \mathrm{~h}$ after $\mathrm{Ni}^{2+}$ application. The $\mathrm{Ni}^{2+}$ uptake rate coefficient was set to $1.17 \times 10^{-8} \mathrm{~mol} \mathrm{~m}^{-2} \mathrm{~s}^{-1}$

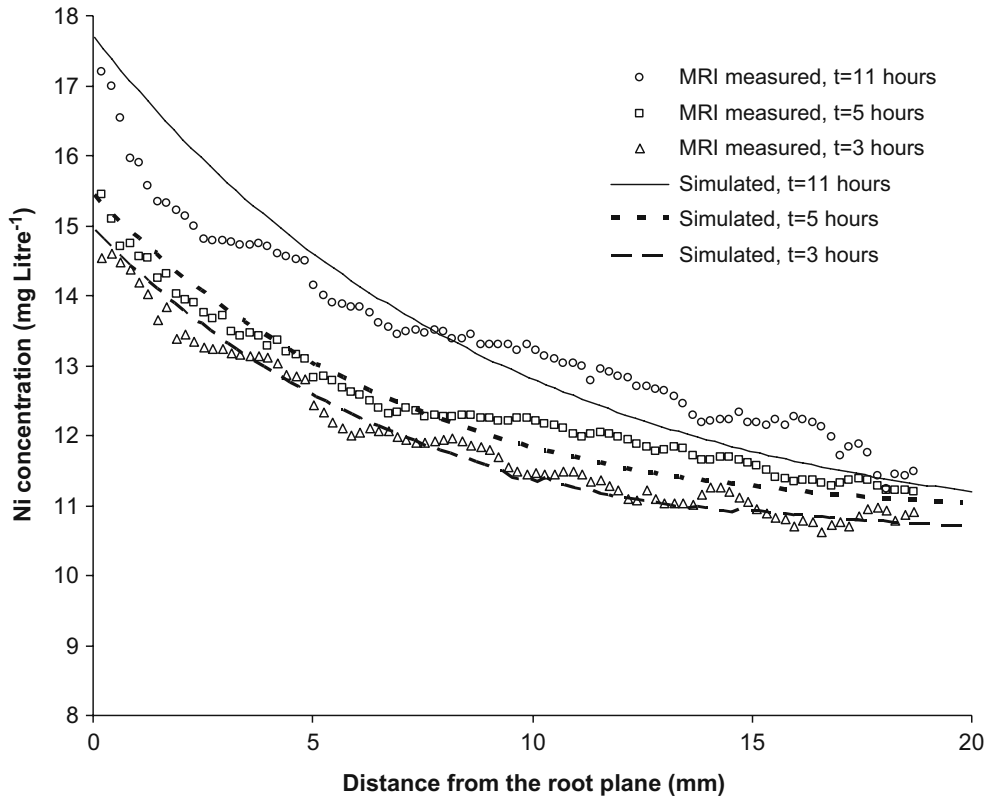


Fig. 7 The MRI-measured and simulated $\mathrm{Ni}^{2+}$ concentration profile as a function of distance from root surface at $t=11 \mathrm{~h}$ and the simulation results assuming a transpiration rate of 45 and $85 \%$ of the measured transpiration for Berkheya coddii

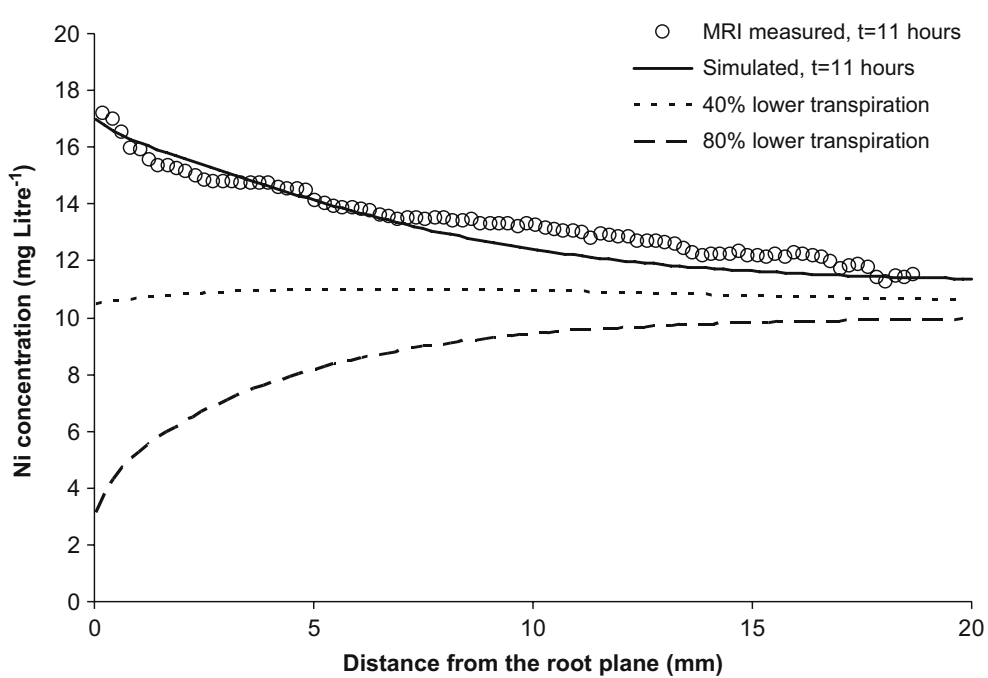

Figure 9 shows simulated $\mathrm{Ni}^{2+}$ gradients assuming various initial concentration of $\mathrm{Ni}^{2+}$ in soil solution. The $\mathrm{Ni}^{2+}$ gradients were normalized by dividing by the initial $\mathrm{Ni}^{2+}$ concentrations. Using the same input parameters but varying the initial concentration of $\mathrm{Ni}^{2+}$, resulted in different $\mathrm{Ni}^{2+}$ gradients. For $\mathrm{Ni}^{2+}$ initial concentration of $1 \mathrm{mg} \mathrm{l}^{-1}$, the simulated accumulation pattern was gentler than $10 \mathrm{mg} \mathrm{l}^{-1}$. A slight $\mathrm{Ni}^{2+}$ depletion was obtained for $\mathrm{Ni}^{2+}$ initial concentration of $0.1 \mathrm{mg} \mathrm{l}^{-1}$ while assuming a $\mathrm{Ni}^{2+}$ initial concentration of $0.01 \mathrm{mg} \mathrm{l}^{-1}$ resulted in an exhaustion of $\mathrm{Ni}^{2+}$ in the soil solution in the rhizosphere.

\section{Discussion}

The experimental set-up that we used here is a compromise between a MRI-suited medium and a realistic soil-root system. Nevertheless, MRI proved to be useful to study the dynamics of $\mathrm{Ni}^{2+}$ in the vicinity of the roots of hyperaccumulator plants non-destructively. Here for the first time we obtained $\mathrm{Ni}^{2+}$ concentration maps in the root zone of Berkheya coddii with spatial resolution of $0.21 \mathrm{~mm}$ and temporal resolution of only minutes. There is a gap of knowledge about the uptake behaviour of hyperaccumulator plants. There is also
Fig. 8 Simulated $\mathrm{Ni}^{2+}$ concentration profiles as a function of distance from root surface for four different uptake rate coefficients. All other parameters were kept constant

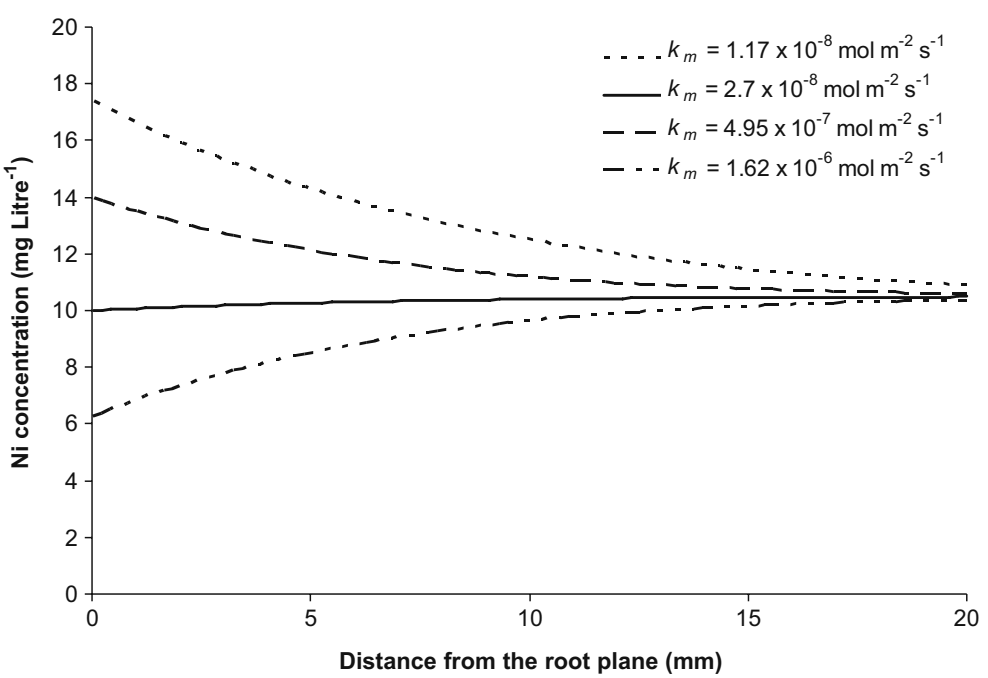




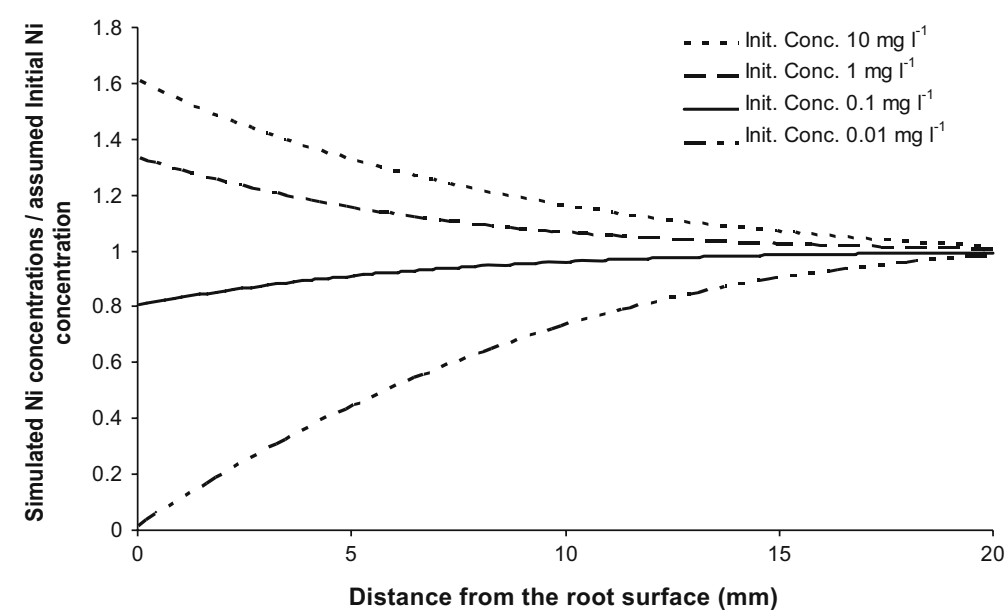

Fig. 9 Simulated $\mathrm{Ni}^{2+}$ concentration profile as a function of distance from root surface assuming various initial concentrations. $\mathrm{Ni}^{2+}$ concentration profiles were normalized by dividing by the corresponding initial concentration. All other parameters were kept constant

desire for a non-destructive non-invasive technique to study rhizosphere processes. Therefore, MRI can be used to study the uptake behaviour of hyperaccumulator plants. However there are several limitations in applying MRI to a real soil-root system. The porous medium that was used here is chemically inert, while the real soil-root system requires accounting for interactions between the ions in the soil solution and the solid phase. MRI was proved to suffer from fading of SNR in unsaturated conditions. The resulting SNR from the unsaturated zone of the rhizobox showed a considerable degree of random variability in unsaturated conditions, representing variations of water content but also water distribution in the pore space. Our images had a spatial resolution of $0.21 \times 0.21 \mathrm{~mm}$, a slice thickness of $15 \mathrm{~mm}$, and therefore, a voxel size of $0.21 \times 0.21 \times$ $15 \mathrm{~mm}$. Considering the size of the glass beads $(0.4$ $1.0 \mathrm{~mm}$ ), the voxel's space was occupied by a random combination of beads and pore space that affects the relaxation behaviour and SNR. This holds true for even a completely water-saturated porous medium. Since each voxel contains a specific combination of beads, pore space and water content, it returns a specific SNR, which is different from the neighbouring voxels. This could be an advantage or disadvantage depending on the type of the information that is desired. For example, it clearly reflects the heterogeneity of the system. However, it makes any averaging of the MRI signal, $T_{1}$ or concentrations along a profile deviating. Therefore, the voxel size should be optimised based on the aims of the study. Additionally, the variability of water saturation of the intra-grain pore space might affect the $T_{1}$ relaxation behaviour and SNR (Fig. 5). To correct for this, separate calibration curves would be needed for given water contents and possibly also for different water distribution patterns in the pore space. Another shortcoming is the detection limit of MRI for $\mathrm{Ni}^{2+}$ concentration. We could resolve $\mathrm{Ni}^{2+}$ concentrations down to $1 \mathrm{mg} \mathrm{l}^{-1}$. However, this could be improved at the cost of measurement time and spatial resolution.

The increase in $\mathrm{Ni}^{2+}$ concentration towards the root surface (Fig. 4) means that $\mathrm{Ni}^{2+}$ uptake by roots was not limited by diffusion at these relatively high soluble $\mathrm{Ni}^{2+}$ concentrations. Our result suggests that Berkheya coddii can hyperaccumulate Ni in soils with high Ni concentration in soil solution without actively solubilizing $\mathrm{Ni}$ in the root zone. Therefore, the $\mathrm{Ni}$ diffusion in such soils is not the limiting factor for phytoremediation or phytomining purposes. This might be the case in serpentine soils, where Berkheya coddii is native.

Our simulation results showed that metal concentration gradient in the rhizosphere is very dynamic and is controlled by plant's transpiration rate, metal uptake rate and the metal concentration in the soil solution (Figs. 7, 8, and 9). Diurnal change of transpiration is expected to affect the gradients of metals in the rhizosphere. For example for metals and ions that are abundant in soil solution, a positive gradient might develop at the peak of transpiration rate in midday, while it may disappear by reduction in transpiration in the evenings. Metal gradients in the rhizosphere of various plant species with various uptake properties might be different. Plants with 
lower metal uptake-rate are more likely to experience accumulation of metal near their roots.

The total Nickel extractable by $1 M$ ammonium acetate was found to reach concentrations up to $12 \mathrm{mg} \mathrm{l}^{-1}$ in serpentine soils in Berkheya coddii's native environment (Robinson et al. 1997), while it is usually present in much lower concentrations in other soils. The metal concentration profile in the rhizosphere of the plants growing in highly contaminated soils might differ from those in less contaminated soils. Figure 9 illustrates that an initial concentration of $10 \mathrm{mg} \mathrm{l}^{-1}$ resulted in an accumulation pattern, while an initial concentration of less than $0.1 \mathrm{mg} \mathrm{l}^{-1}$ resulted in an extensive depletion of $\mathrm{Ni}^{2+}$ adjacent to the roots. This shows that a depletion of $\mathrm{Ni}^{2+}$ is expected at low dissolved concentrations of $\mathrm{Ni}^{2+}$ in soil solution, if the uptake rate stays unchanged. However, the interactions between the sorbed and soluble Ni in soil needs to be considered. Puschenreiter et al. (2005) studied $\mathrm{Ni}$ in the rhizosphere of the hyperaccumulator Thlaspi goesingense and reported an accumulation of $\mathrm{Ni}$ in soil solution at the root surface but a depletion of labile Ni towards the roots. Their results suggests that hyperaccumulation of $\mathrm{Ni}$ (at much lower Ni concentrations in soil solution than in our case) is influenced by the interactions between $\mathrm{Ni}$ in soil solution and the soil solid-phase. They also highlighted the importance of root-induced changes in the rhizosphere. Local changes in dissolved organic matter and $\mathrm{pH}$ due to root exudates can influence the speciation of metals in the soil solution and their mobilisation from the soil solid-phase. These interactions were not accounted for here, neither in the MRI measurements nor in the modelling.

In conclusion, this study shows that even though the real soil-root system was to some extent compromised, combination of MRI and numerical modelling could provide some valuable insight into the dynamic of metals in the root-soil interface. $\mathrm{Ni}$ uptake by Berkheya coddii was shown to be passive and $\mathrm{Ni}$ was partly excluded from the roots and accumulated in the rhizosphere at the concentration range that was used here. The accumulation or depletion of $\mathrm{Ni}^{2+}$ in the root zone of hyperaccumulator plants were demonstrated to be a delicate function of sensitive parameters such as transpiration rate, $\mathrm{Ni}^{2+}$ uptake rate and initial $\mathrm{Ni}^{2+}$ concentration in the soil solution. Our simulation results showed that while decreasing the $\mathrm{Ni}^{2+}$ uptake rate coefficient resulted in accumulation of $\mathrm{Ni}^{2+}$ in the rhizosphere, a depletion pattern was developed when the initial $\mathrm{Ni}^{2+}$ or the transpiration rate was reduced.

Acknowledgements This study was funded by the Swiss National Science Foundation. We would like to thank Reto Treier (IBE, ETH Zurich) for his technical support on $T_{1}$ calculations.

\section{References}

Aggelopoulos CA, Tsakiroglou CD (2007) The longitudinal dispersion coefficient of soils as related to the variability of local permeability. Water Air Soil Poll 185:223-237

Ashworth DJ, Alloway BJ (2004) Soil mobility of sewage sludge-derived dissolved organic matter, copper, nickel and zinc. Environ Pollut 127:137-144

Brooks RR (ed) (1998) Plants that hyperaccumulate heavy metals. CAB International, Wallingford

Brooks RR, Lee J, Reeves RD, Jaffre T (1977) Detection of nickeliferous rocks by analysis of herbarium specimens of indicator plants. J Geochem Explor 7:49-57

Brooks RR, Chambers MF, Nicks LJ, Robinson BH (1998) Phytomining. Trends Plant Sci 3:359-362

Darrah PR (1993) The rhizosphere and plant nutrition-a quantitative approach. Plant Soil 156:1-20

Deoni SCL, Rutt BK, Peters TM (2003) Rapid combined T-1 and T-2 mapping using gradient recalled acquisition in the steady state. Magn Reson Med 49:515-526

Gregory PJ, Hinsinger P (1999) New approaches to studying chemical and physical changes in the rhizosphere: an overview. Plant Soil 211:1-9

Herrmann KH, Pohlmeier A, Wiese S, Shah NJ, Nitzsche O, Vereecken H (2002) Three-dimensional nickel ion transport through porous media using magnetic resonance imaging. J Environ Qual 31:506-514

Hinsinger P, Plassard C, Jaillard B (2006) Rhizosphere: a new frontier for soil biogeochemistry. J Geochem Explor 88:210-213

Kraemer U, CotterHowells JD, Charnock JM, Baker AJM, Smith JAC (1996) Free histidine as a metal chelator in plants that accumulate nickel. Nature 379:635-638

Li YM, Chaney R, Brewer E, Roseberg R, Angle JS, Baker A, Reeves R, Nelkin J (2003) Development of a technology for commercial phytoextraction of nickel: economic and technical considerations. Plant Soil 249:107-115

Lombi E, Zhao FJ, McGrath SP, Young SD, Sacchi GA (2001) Physiological evidence for a high-affinity cadmium transporter highly expressed in a Thlaspi caerulescens ecotype. New Phytol 149:53-60

Mayer KU, Frind EO, Blowes DW (2002) Multicomponent reactive transport modeling in variably saturated porous media using a generalized formulation for kinetically controlled reactions. Water Resour Res 38:1174-1194

McNear DH, Peltier E, Everhart J, Chaney RL, Sutton S, Newville M, Rivers M, Sparks DL (2005) Application of quantitative fluorescence and absorption-edge computed microtomography to image metal compartmentalization in Alyssum murale. Environ Sci Technol 39:2210-2218 
Moradi AB, Oswald SE, Massner JA, Pruessmann KP, Robinson BH, Schulin R (2008) Magnetic Resonance Imaging methods to reveal the real-time distribution of nickel in porous media. Eur J Soil Sci 59:476-485

Pierret A, Doussan C, Garrigues E, Mc Kirby J (2003) Observing plant roots in their environment: current imaging options and specific contribution of twodimensional approaches. Agronomie 23:471-479

Puschenreiter M, Schnepf A, Millan IM, Fitz WJ, Horak O, Klepp J, Schrefl T, Lombi E, Wenzel WW (2005) Changes of $\mathrm{Ni}$ biogeochemistry in the rhizosphere of the hyperaccumulator Thlaspi goesingense. Plant Soil 271:205-218

Robinson BH, Brooks RR, Howes AW, Kirkman JH, Gregg PEH (1997) The potential of the high-biomass nickel hyperaccumulator Berkheya coddii for phytoremediation and phytomining. J Geochem Explor 60:115-126

Robinson BH, Brooks RR, Gregg PEH, Kirkman JH (1999) The nickel phytoextraction potential of some ultramafic soils as determined by sequential extraction. Geoderma 87:293-304

Tinker PB, Nye PH (2000) Solute movement in the rhizosphere. Oxford University Press, New York
Treier R, Steingoetter A, Fried M, Schwizer W, Boesiger P (2007) Optimized and combined T-1 and B-1 mapping technique for fast and accurate T-1 quantification in contrast-enhanced abdominal MRI. Magn Reson Med 57:568-576

Wenzel WW (2005) Rhizosphere conference. J Environ Qual 34:2156-2156

Wenzel WW, Wieshammer G, Fitz WJ, Puschenreiter M (2001) Novel rhizobox design to assess rhizosphere characteristics at high spatial resolution. Plant Soil 237:37-45

Whiting SN, Broadley MR, White PJ (2003) Applying a solute transfer model to phytoextraction: zinc acquisition by Thlaspi caerulescens. Plant Soil 249:45-56

Wosten JHM, Vangenuchten MT (1988) Using texture and other soil properties to predict the unsaturated soil hydraulic functions. Soil Sci Soc Am J 52:1762-1770

Zhao FJ, Hamon RE, Lombi E, McLaughlin MJ, McGrath SP (2002) Characteristics of cadmium uptake in two contrasting ecotypes of the hyperaccumulator Thlaspi caerulescens. J Exp Bot 53:535-543

Zhao FJ, Jiang RF, Dunham SJ, McGrath SP (2006) Cadmium uptake, translocation and tolerance in the hyperaccumulator Arabidopsis halleri. New Phytol 172:646-654 